\title{
Treatment of Osteopathology of the Jaws Associated with Bone Resorption Inhibitors: An 8-Year Single-Centre Experience
}

\section{Jacobsen $\mathbf{C}^{1 *}$, Zemann $\mathbf{W}^{1}$, Obwegeser JA', Seifert $\mathbf{B}^{2}$, Gratz $\mathbf{K W}^{1}$ and Metzler $\mathbf{P}^{1}$}

${ }^{1}$ Department of Craniomaxillofacial Surgery, University Hospital of Zurich, Switzerland

2Insitute of Biostatistics, University of Zurich, Switzerland

\begin{abstract}
Since the discovery that treatment with oral or intravenous bisphosphonate causes bisphosphonate-related osteonecrosis of the jaws (BRONJ), numerous articles on this disease have been published. The number of causative agents was extended after intervention of RANKL-inhibitor denosumab, which has been shown to be as well associated with osteopathology of the jaws. Nonetheless, knowledge of the specific pathogenesis of this clinical entity remains lacking, and no standardized treatment protocols are available. Therefore, despite the vast literature on osteonecrosis of the jaws, there is still considerable ambiguity regarding the optimum treatment of this disease, especially among general practitioners who deal increasingly with bisphosphonates.
\end{abstract}

Keywords: Bisphosphonate-related osteonecrosis; Osteopathology; Computed tomography

\section{Introduction}

Since the discovery that treatment with oral or intravenous bisphosphonate causes Bisphosphonate-Related Osteonecrosis of the Jaws (BRONJ), numerous articles on this disease have been published. The number of causing agents was extended after intervention of RANKL-inhibitor denosumab, which has been shown to be as well associated with osteopathology of the jaws [1]. With increasing experience, knowledge of the epidemiology, possible risk factors, optimal diagnostic approach, and preventive measures has increased. Risk factors include bone-resorption inhibitors, especially their cumulative dose, dental extraction, implant insertion, pressure ulcers, and dental infection [2-4]. To diagnose the extent of the lesion and plan the optimal treatment, Computed Tomography (CT), cone-beam CT, and Magnetic Resonance Imaging (MRI) are helpful [5,6].

Nonetheless, knowledge of the specific pathogenesis of this clinical entity remains lacking, and no standardized treatment protocols are available. Initially, it was experienced only that BRONJ was extremely therapy resistant. Following reports on early wound breakdown after surgical therapy and the failure of wound healing, a conservative approach to treating patients with BRONJ had been accepted globally since $2003[7,8]$.

However, there have been few reports recently of successful cure of patients with conservative or surgical strategies. These treatment strategies ranged from non-invasive to very invasive surgical methods involving resection and reconstruction using microvascularised bone flaps. For example, Wilde et al. [8] reported an $88 \%$ remission rate using surgical resection and bilayer wound closure. Others, for example, Eckardt et al. [9] reports surgical therapy in oncology patients as a "challenging problem". Therefore, despite the vast literature on osteonecrosis of the jaws, there is still considerable ambiguity regarding the optimum treatment of this disease, especially among general practitioners who deal increasingly with bisphosphonates.

This work sought to establish an effective, as restrictive as possible treatment protocol for patients with osteopathology of the jaws associated with antiresorptive therapy based on our 8 years of experience treating BRONJ patients in a special clinic.

\section{Material and Methods}

To better understand the problems and establish appropriate
BRONJ treatment strategies, our patients were treated and followed in a special outpatient clinic, and all cases were documented carefully. In total, 111 patients were referred to this clinic. All patients were diagnosed clinically and by diagnostic imaging or histological examinations, depending on the individual case and the patient's general health.

\section{Treatment}

Initially, our clinic administered primarily conservative treatment. However, we soon realized that conservative treatment increased the frequency of acute infections and consequently the frequency and duration of antibiotic treatment. In addition, the quality of life of patients with infected exposed bone was very poor because of pain, halitosis, antibiotic side effects, and the need for numerous visits to the dentist for rinsing of the affected jawbone. Most patients were unable to keep the exposed bone clean and infection-free because of their reduced dexterity due to poor general health or age. Therefore, minor surgical therapy was performed to a greater extent, depending on the lesion and the patient's condition. The basic intent was to remove the infected, necrotic bone, smooth the healthy underlying bone, and attain tight wound closure with vertical, resorbable mattress sutures, thus preventing dead space. In the posterior maxillary region, with involvement of the maxillary sinus and oroantral fistula, the buccal fat pad was mobilized and used for soft tissue closure. Around 2007, careful rinsing with antibiotic solution (neomycin) before wound closure was started. The patients were followed closely until overlying mucosa had healed completely. The sutures were removed 10 days after surgery. In patients undergoing conservative treatment, follow-up visits were scheduled on a weekly to monthly basis, depending on the individual situation.

${ }^{*}$ Corresponding author: Dr. Jacobsen C, M.D, D.M.D, Department of Craniomaxillofacial Surgery, University Hospital of Zurich, Switzerland, Tel: +4144 2555058; Fax: +41 44255 8989; E-mail: christine.jacobsen@usz.ch

Received August 10, 2012; Accepted October 18, 2012; Published October 20, 2012

Citation: Jacobsen C, Zemann W, Obwegeser JA, Seifert B, Gratz KW, et al. (2012) Treatment of Osteopathology of the Jaws Associated with Bone Resorption Inhibitors: An 8-Year Single-Centre Experience. Dentistry 2:146. doi:10.4172/2161. 1122.1000146

Copyright: (C) 2012 Jacobsen C, et al. This is an open-access article distributed under the terms of the Creative Commons Attribution License, which permits unrestricted use, distribution, and reproduction in any medium, provided the original author and source are credited. 
We recorded the gender, age, underlying disease, drug regimen, local risk factors, symptoms, and clinical picture of all patients. In addition, the diagnostic measures, treatment protocol, and outcome measures of patients treated surgically were recorded.

When the clinic was opened, conservative treatment was chosen because our protocol included avoidance of invasive measures to prevent progression. Subsequently, it was adopted mainly in cases of poor general health or if the patient refused surgery.

Malignant cells were found in the histological specimens from two patients. Therefore, they were excluded from the study. In total, the study included 109 patients undergoing either conservative or surgical treatment.

All specimens were primarily obtained for medical purposes, with the informed consent of the patients. The study design fulfills the guidelines of the Declaration of Helsinki regarding ethical principles for medical research involving human subjects.

\section{Statistical analysis}

For statistical analysis of binary variables, Fisher's exact test was performed with a two-sided $p$-value $<0.05$ indicating significance. The Mann-Whitney $U$-test was used to analyze continuous variables, such as age and the duration of therapy. The statistical analysis was performed using IBM SPSS Statistics, version $19^{\circ}$ (SPSS Inc., Chicago, IL).

\section{Results}

\section{General factors}

In total, 109 patients were treated and followed at our clinic: $58 \%$ were treated surgically and $42 \%$ conservatively. Of the patients treated surgically, $71 \%$ were female, whereas in the patients treated conservatively, $67 \%$ were female. The mean ages of the patients treated surgically and conservatively were 68 (range 43-94 years) and 65 (range 37-89 years) years, respectively.

\section{Underlying disease}

Approximately three-quarters $(n=82)$ of the patients had underlying malignant disease, while the remaining quarter $(n=27)$ had osteoporosis. Of the patients with malignant disease, almost half had breast cancer, regardless of whether the BRONJ was treated surgically or conservatively. Multiple myeloma was present in $26 \%$ of the conservatively treated group and $32 \%$ of the surgically treated group; other patients suffered from prostate cancer, lymphoma, lung carcinoma, bladder cancer, malignant melanoma, renal cell carcinoma, and carcinoma of the thyroid.

\begin{tabular}{|c|c|c|c|c|c|c|c|c|}
\hline & \multicolumn{4}{|c|}{ Surgery $n(\%)$} & \multicolumn{4}{|c|}{ Conservative $\mathrm{n}(\%)$} \\
\hline & $\begin{array}{l}\text { non malig- } \\
\text { nancy }\end{array}$ & $\begin{array}{l}\text { malignant } \\
\text { disease }\end{array}$ & n.i.1 & total & non malignancy & $\begin{array}{l}\text { malignant } \\
\text { disease }\end{array}$ & n.i.1 & total \\
\hline & $19(30)$ & $44(70)$ & & $63(58)$ & $8(17)$ & $38(83)$ & & $46(42)$ \\
\hline mean age (years) & & & & 68 & & & & 65 \\
\hline female & & & & $45(71)$ & & & & $31(67)$ \\
\hline \multicolumn{9}{|l|}{ Underlying disease } \\
\hline Multiple Myeloma & & $14(32)$ & & & & $10(26)$ & & \\
\hline Breast cancer* & & 21(48) & & & & $18(47)$ & & \\
\hline prostate cancer & & $6(14)$ & & & & $4(11)$ & & \\
\hline others* & & $4^{*}(9)$ & & & & $6(16)$ & & \\
\hline \multicolumn{9}{|l|}{ drug regimen* } \\
\hline zoledronic acid & $4^{* * *}$ & 36 & & $40(63)$ & 1 & 32 & & $33(72)$ \\
\hline pamidronate & 3 & 8 & & 11 & 2 & 5 & & 7 \\
\hline alendronate & 7 & 1 & & 8 & 4 & 1 & & 5 \\
\hline ibandronate & 9 & 1 & & 10 & 2 & & & 2 \\
\hline others & 0 & 7 & & 7 & 1 & 1 & & 2 \\
\hline duration of $\mathrm{BP}(\text { months })^{\star *}$ & 43 & 37 & 3 & 39 & 31 & 28 & 9 & 28 \\
\hline \multicolumn{9}{|l|}{ local risk factor } \\
\hline extraction & 11 & 26 & & $37(59)$ & 3 & 25 & & 28(61) \\
\hline prosthesis & 5 & 9 & & 14 & 2 & 2 & & 4 \\
\hline implants & 3 & 6 & & 9 & 3 & 0 & & 3 \\
\hline periodontal disease & 1 & 5 & & 6 & 0 & 3 & & 3 \\
\hline Dental infection & 4 & 10 & & $14(22)$ & 2 & 10 & & 22(26) \\
\hline duration of PB stopp (months) & 5 & 6 & & 6 & & & & \\
\hline general anaesthesia & & & & $55(87)$ & & & & \\
\hline local anaesthesia & & & & 8 & & & & \\
\hline \multicolumn{9}{|l|}{ outcome } \\
\hline complete remission & $18(95)$ & $31(70)$ & & 49(78) & & & & \\
\hline partial remission & 1 & 7 & & $8(13)$ & & & & \\
\hline no improvement & 0 & 6 & & 6 & & & & \\
\hline \multicolumn{9}{|l|}{${ }^{1}$ no information } \\
\hline \multicolumn{9}{|c|}{ *patient may belong to more than one group } \\
\hline \multicolumn{9}{|l|}{${ }^{* *}$ until the first presentation } \\
\hline *** all patients received zoledror & year & & & & & & & \\
\hline
\end{tabular}

Table 1: Display of descriptive statistics for patients, subdivided into type of treatment and underlying disease. 


\section{Drug regimen}

The patients had been treated previously with zoledronic acid, pamidronate, alendronate, and ibandronate, in order of decreasing frequency (Table 1). Some patients had change of drug regimen during the course of underlying disease. In the group of conservatively treated patients, three women with osteoporosis were treated with intravenous therapy with either zoledronic acid or pamidronate in a monthly course.

\section{Local risk factor and clinical presentation}

The majority of patients presented with a non-healing socket after tooth extraction. In six patients of the surgically treated group dental implants have been placed, although patients had underlying malignant disease and intravenous bisphosphonate treatment at the time of implant placement. A considerable amount of patients had dental infection (e.g. periapically) that was clearly related to the area of BRONJ.

Most patients presented with pain, but all patients had signs of inflammation. In total 77 (70\%) presented with exposed bone, whereas the other patients had apparent BRONJ, without exposed bone. Eighteen patients $17 \%$ had a sinus tract and 23 patients $21 \%$ presented with an abscess as the first clinical sign.

\section{Treatment}

In total 64 patients were treated surgically, whereas 46 patients had conservative treatment (Figures 1a-1c). The mean time from the first appointment at our clinic to surgical intervention was 7.4 months. Initially, patients were operated on soon after their first appointment in the clinic; subsequently, however, we stop bisphosphonate treatment for 3-6 months before the intervention (Figures 2a-2c).

Five patients underwent more aggressive treatment: three had reconstruction plates inserted; one received a microsurgical revascularized fibula flap; and one underwent resection and immediate reconstruction augmented with autologous iliac crest bone. Complete remission was achieved in four of these patients. The remaining

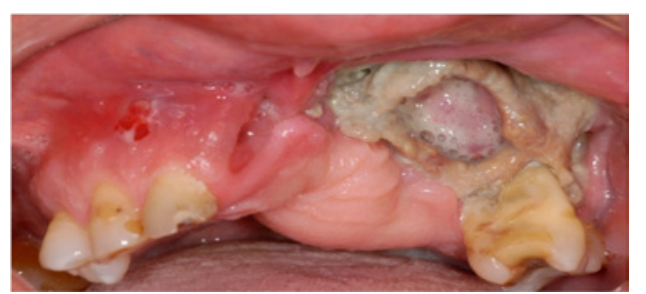

Figure 1a: Male patient with palliative situation due to bladder carcinoma with a massive osteonecrosis of the entire maxilla and centrolateral midface on the left side.

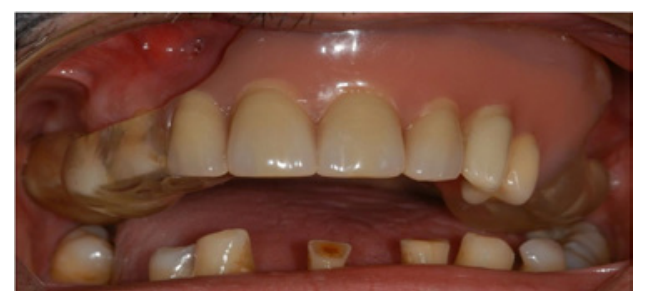

Figure 1b: Situation after conservative treatment with antibiotics and smoothening of bony edges.

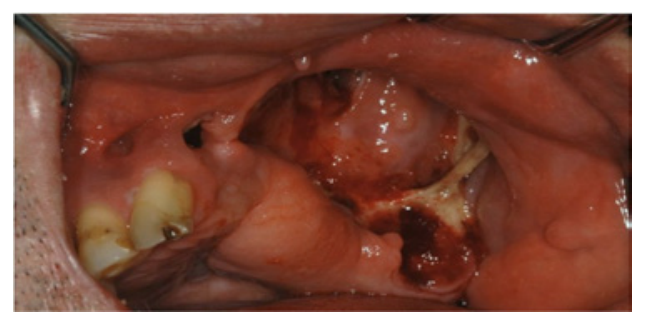

Figure 1c: Dental rehabilitation with individualized obturator prosthesis. Function and speech could be rehabilitated.
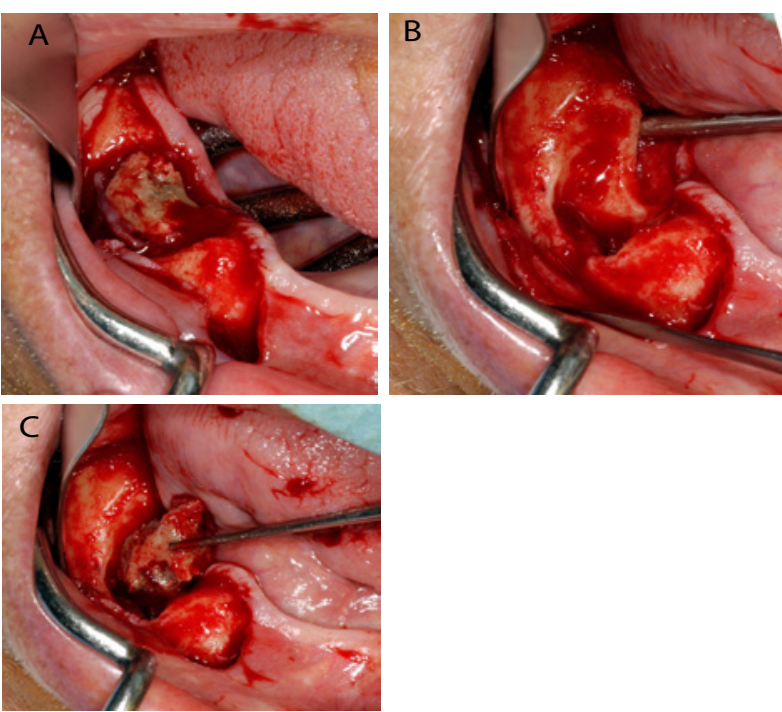

Figure 2: Male patient with underlying multiple myeloma shows sequester formation after drug holiday with subsequent sequestrectomy.

patient showed no improvement; indeed, their bone destruction and underlying multiple myeloma progressed.

\section{Histological examination}

Histological examinations of the intraoperative bone specimens of 54 patients were performed. Partially avital bone with signs of acute and chronic inflammation was found in all patients. In 38 patients, Actinomyces granules were found. In the remaining 16 patients, the biopsies were not specifically analyzed for Actinomyces because this examination was first requested in 2008.

\section{Outcomes of surgical treatment}

In total, $78 \%$ of the patients treated surgically had complete resolution of their disease, and $12 \%$ had partial remission, i.e, symptom improvement and less exposed bone. No improvement was seen in six patients, four of whom underwent surgery in 2003-2006 without stopping bisphosphonates and using a regressive surgical approach. Two were in poor general condition and receiving additional concomitant chemotherapy.

\section{Influencing factors for treatment outcome}

Of the osteoporosis patients, 95\% achieved complete remission versus $70 \%$ of those with malignant disease. Non-malignant underlying disease is a significant $(\mathrm{p}=0.047)$ predictor of achievement of complete remission $(\mathrm{p}=0.047)$.

After 2008, with use of an increasingly standardized protocol, the 


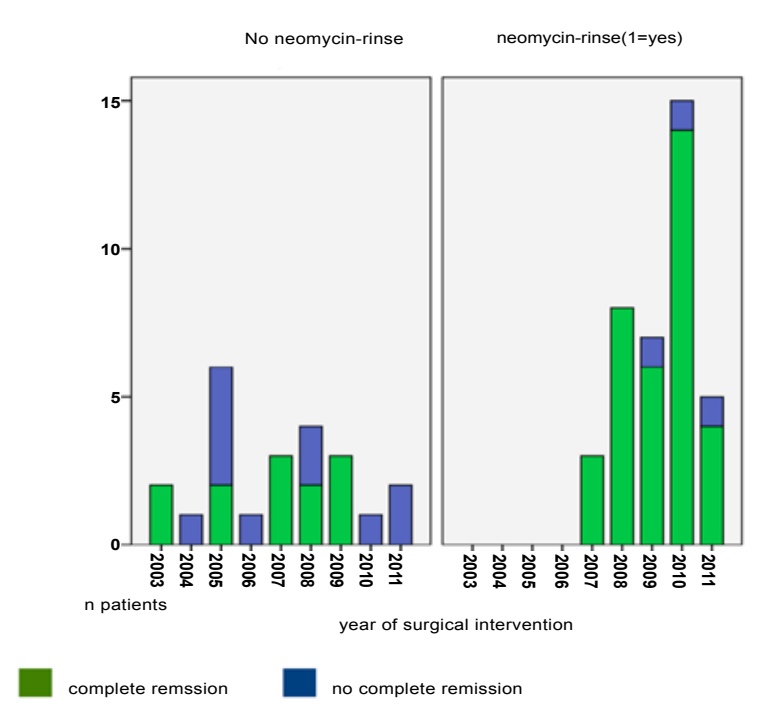

Figure 3: Display of number of patients with complete remission during the years 2003- 2011 subdivided in: with-, or without rinsing with neomycin.

rate of complete remission increased to $83 \%$ (39 of 47). Intraoperative rinsing with antibiotic solution seemed to be one important cause of this. Thirty five patients underwent intraoperative rinsing with antibiotic solution (neomycin). Rinsing was also a significant ( $\mathrm{p}=0.001$ ) predictor of a better outcome (Figure 3 ). The outcome was negatively influenced by previous treatment with zoledronic acid $(p=0.05)$ and first presentation with an abscess $(\mathrm{p}=0.035)$. No other variables had a significant influence on the outcome, including age, drug type, drug duration, local factors, and localization.

\section{Outcomes of conservatively treated patients}

Of the patients treated conservatively, five achieved complete remission and all exhibited symptomatic improvement. Sixteen patients in this group died soon after their diagnosis of BRONJ, due to their underlying disease and poor general health.

\section{Discussion}

Osteopathology related to therapy with bone resorption inhibitors, mainly bisphosphonates, is reported frequently. Nevertheless, our knowledge of the pathophysiology is poor and there is no effective, evidence-based, standardized therapy protocol. This study analyzed the development of an effective therapy strategy for patients with osteopathology seen in a single centre over the last 8 years. Almost half of the patients were treated conservatively, although the proportion decreased over time. Initially, our treatment protocol for BRONJ was mainly conservative, based on our experience, reports of therapy resistance of this disease, and general recommendations to avoid surgical treatment. Around 2008, the treatment protocol changed slowly, and patients were increasingly operated on with promising results. In our series, $58 \%$ of the patients underwent surgery; $78 \%$ achieved complete remission and a further $13 \%$ considerable improvement. Four of the six patients with no improvement underwent surgery in the early years, without halting their bisphosphonate therapy. Interestingly, wound breakdown often occurred several weeks after surgery.

In most patients, discontinuing bisphosphonate therapy resulted in sequestra formation after about 6 months, defining the extent of the surgical treatment. In addition, careful rinsing with antibiotic solution before wound closure seemed to improve wound healing significantly.

Successful surgical treatment of BRONJ patients has been reported in the last few years [7]. For example, Wilde et al. [8] performed a similar surgical technique in 20 patients with BRONJ, with a success rate of $88 \%$. However, they did not stop bisphosphonate treatment before surgery in every patient. Similar to our therapeutic access, their access was relatively less invasive, causing comparatively little discomfort or reduction in the patient's quality of life, which is important in the evaluation and treatment of patients with BRONJ. The majority of patients have a long history of symptoms and therapies restricting their quality of life. Therefore, it is extremely important to find the best treatment option based on the patient's history and requirements. Moretti et al. [10] used this argument in their prospective evaluation of a minimally invasive treatment protocol. They advocated their conservative protocol, arguing that surgical intervention was inappropriate in an "already highly compromised" situation. Various factors affected the patients' quality of life: some had poor general health, and Moretti et al. [10] stated that surgery would have been too exhausting and the risk of general anesthesia too high; other patients suffered severely from recurrent infections, halitosis, pain, side effects of antibiotic treatment, and the need to visit a dentist frequently for rinsing. Most patients were unable to keep the BRONJ area clean, although they were "adequately informed, motivated and followed" [10]. In those patients, performance of only minor surgery is important to increase their quality of life. Symptoms such as pain can be controlled with local and systemic antibiotic therapy for a time, although in our experience, a cure can be accomplished only by resection of the infected, necrotic bone. Therefore, the treatment for each patient with osteopathology of the jaws should be assessed individually, considering the local situation (e.g, staging system) and general situation and request of the patient.

Resection of the infected, necrotic bone and soft tissue coverage or reconstruction with various levels of aggressiveness and using different protocols has been described. For example, Curi et al. [11] introduced a protocol involving bone resection and the use of autologous plateletrich plasma, which achieved success in $80 \%$ of 25 patients. Lemound et al. [12] achieved a success rate of $90 \%$ in 20 patients using a myofascial flap. Pautke et al. [13] described a fluorescence-guided bone resection technique with an $85 \%$ success rate in $20 \mathrm{BRONJ}$ lesions at the 4 -week follow-up. We use simple resection of the necrotic bone with soft tissue closure after adequate mobilization or the use of buccal fat, with a similar success rate $(83 \%)$. Interestingly, the success rate of the osteoporosis patients was $95 \%$, compared to $70 \%$ for those with malignant disease. The exception was one female with no drug holiday who had a partial remission. In our experience, two main steps improved wound healing: the discontinuation of bisphosphonates 3-6 months before surgical intervention and extensive rinsing with antibiotic solution before wound closure. After discontinuing bisphosphonate, bone remodeling seemed to restart slowly, and in most cases a sequestrum formed in the affected bone, facilitating surgery. Rinsing with an antibiotic solution before wound closure might act in two ways: as simple local antiinfectious treatment and via mechanical cleaning and the elimination of bone chips with bound bisphosphonate, which has a toxic effect on the soft tissues $[14,15]$. Of course, these hypotheses must be verified in prospective studies. Regardless, any surgical intervention should be as minor as possible, placing minimal stress on the patient. Given our promising success rates, extensive surgery with subsequent reconstruction with microsurgical revascularized flaps is an option in 
some patients with no other choice, as mentioned by some authors, [16-18] and was performed in three of our patients.

Interestingly, some authors have reported good remission rates with use of only conservative treatment. For example, van den Wyngaert et al. [19] reported total remission with complete closure of the mucosa in $16(53 \%)$ patients. The length of the exposure to bisphosphonates and stage of BRONJ influenced the healing rate, whereas the cessation of bisphosphonates did not. In their study, five patients had complete remission, although a sequestrum developed and granulation tissue formed on the remaining bone with complete closure of the mucosa in three patients in whom bisphosphonate treatment was stopped, after bone remodeling had restarted. Therefore, cure with conservative treatment only is possible if: 1) the bone starts to remodel and demarcates the necrotic area, 2) the immune system is strong enough to control any local infection enabling tissue granulation, and 3) the area is kept free of infection.

Although our series was small, this study shows the development of an effective treatment protocol at a single centre, based on the stage of local and underlying disease, and the patient's dexterity and quality of life.

Minimally invasive surgical treatment should be favored in patients in suitable general health at least 3 months after discontinuation of bisphosphonate treatment, depending on the underlying disease. In patients with poor general condition, conservative treatment is preferred, possibly with local curettage. Extensive surgical resection and reconstruction should only be reserved for patients with extensive osteopathology and marked discomfort, but in good general health and with a good prognosis.

\section{References}

1. Van den Wyngaert T, Wouters K, Huizing MT, Vermorken JB (2011) RANK ligand inhibition in bone metastatic cancer and risk of osteonecrosis of the jaw (ONJ): non bis in idem? Support Care Cancer 19: 2035-2040.

2. Hoff AO, Toth B, Hu M, Hortobagyi GN, Gagel RF (2011) Epidemiology and risk factors for osteonecrosis of the jaw in cancer patients. Ann N Y Acad Sci 1218: 47-54.

3. Vahtsevanos K, Kyrgidis A, Verrou E, Katodritou E, Triaridis S, et al. (2009) Longitudinal cohort study of risk factors in cancer patients of bisphosphonaterelated osteonecrosis of the jaw. J Clin Oncol 27: 5356-5362.

4. Jacobsen C, Metzler P, Rössle M, Obwegeser J, Zemann W, et al. (2012) Osteopathology induced by bisphosphonates and dental implants: clinical observations. Clin Oral Investig.

5. Arce K, Assael LA, Weissman JL, Markiewicz MR (2009) Imaging findings in bisphosphonate-related osteonecrosis of jaws. J Oral Maxillofac Surg 67: 7584.

6. Morag Y, Morag-Hezroni M, Jamadar DA, Ward BB, Jacobson JA, et al. (2009) Bisphosphonate-related osteonecrosis of the jaw: a pictorial review. Radiographics 29: 1971-1984.

7. Williamson RA (2010) Surgical management of bisphosphonate induced osteonecrosis of the jaws. Int J Oral Maxillofac Surg 39:251-255.

8. Wilde F, Heufelder M, Winter K, Hendricks J, Frerich B, et al. (2011) The role of surgical therapy in the management of intravenous bisphosphonates-related osteonecrosis of the jaw. Oral Surg Oral Med Oral Pathol Oral Radiol Endod 111:153-163.

9. Eckardt AM, Lemound J, Lindhorst D, Rana M, Gellrich NC (2011) Surgical management of bisphosphonate-related osteonecrosis of the jaw in oncologic patients: a challenging problem. Anticancer Res 31: 2313-2318.

10. Moretti F, Pelliccioni GA, Montebugnoli L, Marchetti C (2011) A prospective clinical trial for assessing the efficacy of a minimally invasive protocol in patients with bisphosphonate-associated osteonecrosis of the jaws. Oral Surg Oral Med Oral Pathol Oral Radiol Endod 112:777-782.
11. Curi MM, Cossolin GS, Koga DH, Zardetto C, Christianini S, et al. (2011) Bisphosphonate-related osteonecrosis of the jaws--an initial case series report of treatment combining partial bone resection and autologous platelet-rich plasma. J Oral Maxillofac Surg 69: 2465-2472.

12. Lemound J, Eckardt A, Kokemüller H, von See C, Voss PJ, et al. (2011) Bisphosphonate-associated osteonecrosis of the mandible: reliable soft tissue reconstruction using a local myofascial flap. Clin Oral Investig 16: 1143-1152.

13. Pautke C, Bauer F, Otto S, Tischer T, Steiner T, et al. (2011) Fluorescenceguided bone resection in bisphosphonate-related osteonecrosis of the jaws: first clinical results of a prospective pilot study. J Oral Maxillofac Surg 69: 84-91.

14. Cornish J, Bava U, Callon KE, Bai J, Naot D, et al. (2011) Bone-bound bisphosphonate inhibits growth of adjacent non-bone cells. Bone 49: 710-716.

15. Ravosa MJ, Ning J, Liu Y, Stack MS (2011) Bisphosphonate effects on the behaviour of oral epithelial cells and oral fibroblasts. Arch Oral Biol 56: 491-498.

16. Seth R, Futran ND, Alam DS, Knott PD (2010) Outcomes of vascularized bone graft reconstruction of the mandible in bisphosphonate-related osteonecrosis of the jaws. Laryngoscope 120: 2165-2171.

17. Mücke T, Haarmann S, Wolff KD, Hölzle F (2009) Bisphosphonate related osteonecrosis of the jaws treated by surgical resection and immediate osseous microvascular reconstruction. J Craniomaxillofac Surg 37: 291-297.

18. Nocini PF, Saia G, Bettini G, Ragazzo M, Blandamura S, et al. (2009) Vascularized fibula flap reconstruction of the mandible in bisphosphonaterelated osteonecrosis. Eur J Surg Oncol 35:373-379.

19. Van den Wyngaert T, Claeys T, Huizing MT, Vermorken JB, Fossion E (2009) Initial experience with conservative treatment in cancer patients with osteonecrosis of the jaw (ONJ) and predictors of outcome. Ann Oncol 20: 331 336. 